For reprint orders, please contact: reprints@future-science.com

\title{
Corrigendum
}

\section{Future Science}

The research article by authors Bashiru Garba \& Bashir Saidu which appeared in the April 2020 issue of Future Science OA 6(7), FSO475 (2020), was published with the author's name being presented incorrectly as 'Bashir Sa'idu'. This has now been corrected to 'Bashir Saidu'.

The editors and the authors of Future Science $O A$ would like to sincerely apologize for any inconvenience or confusion this may have caused our readers. 